Internist 2010 · 51:951-952

DOI 10.1007/s00108-010-2684-4

Online publiziert: 3. Juli 2010

(c) Springer-Verlag 2010

\author{
K. Werdan \\ Universitätsklinik und Poliklinik für Innere Medizin III, Universitätsklinikum \\ der Martin-Luther-Universität Halle-Wittenberg, Halle (Saale)
}

\title{
Internistische Intensivmedizin kardiovaskulärer Notfälle
}

Liebe Leserinnen und Leser dieses Schwerpunktheftes „Kardiovaskuläre Notfälle“!

Vieles hat sich im Bereich der kardiovaskulären Notfall- und Intensivmedizin in letzter Zeit getan: die Revision der Infarktdefinitionen und die verbesserte Infarktfrüherkennung, der Aufbau neuer Organisationsstrukturen wie der „Chest Pain Unit" und die Festigung bereits etablierter Strukturen wie der "Stroke Unit“, ein Paradigmenwandel bei der Endokarditisprophylaxe und -therapie sowie neue Leitlinien in kaum mehr überschaubarer Zahl. Demzufolge ist es wieder einmal an der Zeit, das wesentliche Wissen und die relevanten Neuerungen in einem Themenheft von Der Internist für Sie darzustellen. Und dies haben wir mit Unterstützung namhafter Experten in der vorliegenden Ausgabe versucht.

Akutes Koronarsyndrom, schwere Herzinsuffizienz und kardiogener Schock sowie lebensbedrohliche Herzrhythmusstörungen sind quantitativ die drängendsten Probleme der kardiovaskulären Notfallmedizin, und deshalb wollen wir auch mit diesen Themen beginnen.

- Immer noch stellt das akute Koronarsyndrom die häufigste Todesursache in Deutschland dar.

In ihrem Beitrag „Das akute Koronarsyndrom" geben die Autoren F. Post und T. Münzel aus Mainz prägnante Definitionen der instabilen Angina pectoris, des „Nicht-ST-Hebungsinfarkts“ (NSTEMI) und des „ST-Hebungsinfarkts“ (STEMI), weisen aber auch auf die etwas unscharfe Verwendung des Begriffs „akutes Koronarsyndrom" in der Praxis hin. Neue kardiale Biomarker wie das hochsensitive Troponin bieten die Möglichkeit, einen NSTEMI früher zu erkennen. Bis zur effektiven Umsetzung in der Praxis sind jedoch sicherlich noch offene Fragen zu klären. Vor allem der richtige Zeitpunkt des koronarinterventionellen Vorgehens beim akuten Koronarsyndrom war in letzter Zeit sehr in der Diskussion. Zu dieser Risikostratifizierung wird in dem Beitrag von Post und Münzel klar Stellung genommen, ebenso wie zu wichtigen Änderungen auf dem Gebiet der medikamentösen Therapie. Zunehmende Bedeutung im Interesse einer optimalen Patientenversorgung besitzen strukturierte Versorgungsnetze für den Patienten mit akutem Koronarsyndrom, zu deren Qualitätsmanagement die Autoren mit ihren Empfehlungen zur Etablierung von „Chest Pain Units“ in Deutschland maßgeblichen Anteil haben.

Ein Hinweis sei zu diesem Artikel noch gestattet. Stehen dem Krankenhausarzt in der Notaufnahme alle diagnostischen Wege offen, so hat es der erstversorgende Hausarzt bei der Diagnose „Thoraxschmerz" oft sehr viel schwerer, die wenigen Patienten mit Angina pectoris und v. a. die mit akutem Koronarsyndrom aus der großen Zahl der Patienten mit nichtkardialen Brustkorbschmerzen „herauszufischen“. Hier möchte ich auf die sehr gelungene Leitlinie „Thoraxschmerz“ der
Deutschen Gesellschaft für Allgemeinmedizin hinweisen (heinemae@staff.unimarburg.de).

Auch die akut dekompensierte Herzinsuffizienz (ADHF) ist aufgrund der demographischen Entwicklung ein quantitativ zunehmendes Problem, wie uns allen bewusst ist. Diesem Thema haben sich die Autoren M. Buerke, H. Lemm, M. Russ und A. Schlitt aus Halle (Saale) mit ihrem Beitrag „Diagnostik und Therapie bei akut dekompensierter Herzinsuffizienz und kardiogenem Schock" gewidmet. Die für die Notfallsituation sehr hilfreiche Einteilung in normotensive, hypertensive und hypotensive (inkl. Schock) Form der akut dekompensierten Herzinsuffizienz folgt in etwa der Einteilung der chronischen Herzinsuffizienz in die systolische (Äquivalent: normo- und hypotensive ADHF) und die diastolische Form (Äquivalent: hypertensive ADHF). Wesentlich ist, dass sich aus dieser Einteilung bereits rasch durchführbare Therapiekonzepte ableiten lassen. Ich bin gespannt, ob die in diesem Beitrag vorgeschlagenen Algorithmen für die jeweiligen Formen der akut dekompensierten Herzinsuffizienz bei unseren Lesern Anklang finden werden.

In seinem Beitrag „Vital bedrohliche brady- und tachykarde Herzrhythmusstörungen“" stellt H.-J. Trappe aus Herne das „5-A-Konzept“ zur antiarrhythmischen Akuttherapie vor, bestehend aus den 5 Medikamenten Adenosin, Adrenalin, Ajmalin, Amiodaron und Atropin zur Behandlung tachykarder und bradykar- 
der Rhythmusstörungen sowie zur Therapie der Asystolie. Ergänzende „B-, Cund D-Strategien“ sind $\beta$-Blocker, Cardioversion und Defibrillation. Das Konzept der ${ }_{5}$ A“ erlaubt nach Aussage des Autors eine sichere und effektive Therapie, und mit der „B-, C- und D-Strategie“ zusammen würden sich alle Notfallsituationen, welche durch Arrhythmien bedingt sind, adäquat behandeln lassen. Der Leser darf also gespannt sein!

Häufig nur mit langer Latenz diagnostiziert und im Gefährdungsgrad unterschätzt wird die Endokarditis. In ihrem Beitrag „Endokarditis“ präsentieren die Autoren B. Plicht,R.-A. Jánosi,T. Buck und R. Erbel aus Essen zu Beginn einen kniffligen Fall und zeigen anhand dieser Kasuistik die „Fallen“ und Schwierigkeiten bei der Diagnosestellung und Behandlung dieses lebensbedrohlichen Krankheitsbilds auf. Die Patientin konnte nach 8 Wochen in eine kardiologische Rehabilitationsklinik weiterverlegt werden, allerdings mit persistierender Niereninsuffizienz - ein wahrhaft nicht einfacher Krankheitsverlauf! Zu Recht betonen die Autoren die Notwendigkeit der unmittelbaren Abnahme von Blutkulturen noch vor Beginn der Antibiotikatherapie und die rasche Durchführung einer Echokardiographie durch einen sehr erfahrenen Untersucher. Meist ist diesbezüglich eine transthorakale Untersuchung allerdings nicht ausreichend, darauf sollt man sich einstellen!

Die entscheidende Frage bei der Lungenembolie ist meines Erachtens nicht die, ob der Patient lysiert werden muss, was ja nur selten der Fall ist. Die viel problematischere Frage ist: Wie gefährdet ist der Patient, auch wenn er nicht im Schock ist? Kann ich den Patienten mit akuter Lungenembolie auf die Allgemeinstation legen oder muss ich ihn noch weiter auf der Intensivstation überwachen? Dieser Frage widmet sich S. Schellong aus Dresden in seinem Beitrag „Der intensivpflichtige Patient mit Lungenembolie oder arteriellem Gefäßnotfall“, denn auch ein Patient ohne Kreislaufinstabilität ist gefährdet, wenn er eine Rechtsherzbelastung zeigt. Insofern ist seine Empfehlung, die Rechtsherzbelastung bei Patienten mit Lungenembolie möglichst gut zu definieren, von eminenter Bedeutung. Wir selbst haben dabei mit dem „Wacker-Score“ sehr gute Erfahrungen gemacht [1], auch wenn dieser Vorschlag keinen Eingang in die Lungenembolieleitlinien der Europäischen Kardiologischen Gesellschaft gefunden hat. Liegt der Wacker-Score bei $\geq 2,25$, so ist der Patient aufgrund seiner Rechtsherzbelastung weiterhin akut gefährdet, auch wenn er sich klinisch nicht im Schock präsentiert. Aber nicht nur die Lungenembolie ist Thema des Beitrags von S. Schellong, sondern auch die akute Extremitätenischämie, die akrale Läsion und die nicht seltenen iatrogenen Gefäßschäden.

\section{- Die Langzeitfolgen eines \\ Schlaganfalls können desaströs sein.}

Umso wichtiger ist es, vermeidbare Schäden durch eine optimale Akuttherapie zu verhindern, wie die Autoren C. Lichy und W. Hacke aus Heidelberg in ihrem Beitrag "Schlaganfall“ betonen: Der Patient mit akutem Schlaganfall ist wirklich als vorrangiger Notfall anzusehen, und auch hier ist - ebenso wie beim Herzinfarkt - das rasche Handeln entscheidend („time is brain“). Gleiches gilt wegen der hohen Rezidivgefahr in den ersten $48 \mathrm{~h}$ auch für $\mathrm{Pa}$ tienten mit ,transienter ischämischer Attacke" (TIA). Eine unverzügliche Schnittbildgebung ist zur Differenzierung von ursächlicher Ischämie oder Hämorrhagie als erster diagnostischer Schritt indiziert, dem idealer Weise bei akuter zerebraler Ischämie mit behinderndem Defizit die unverzügliche Wiederherstellung der zerebralen Perfusion als Therapieprinzip zu folgen hat. Die Autoren geben auch ein klares Statement dafür ab, dass alle akuten Schlaganfallpatienten eine spezialisierte, multidisziplinäre Versorgung benötigen, wie sie auf einer Stroke Unit angeboten werden kann.

Liebe Leserinnen und Leser, kardiovaskuläre Notfälle haben viele Gesichter! Autoren und Herausgeber haben versucht, einige charakteristische Notfälle für Sie abzubilden. Wir hoffen, Ihnen damit einige aktuelle und praxisrelevante kardiovaskuläre Notfallsituationen und deren Handhabung vermittelt zu haben.
Eine informative Lektüre wünscht Ihnen

Ihr

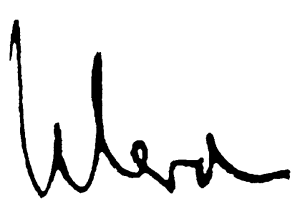

K. Werdan

\section{Korrespondenzadresse \\ Prof. Dr. K. Werdan}

Universitätsklinik und Poliklinik für Innere Medizin III, Universitätsklinikum Halle (Saale) der Martin-Luther-Universität Halle-Wittenberg Ernst-Grube-Straße 40, 06120 Halle/Saale karl.werdan@medizin-uni-halle.de

\section{Literatur}

1. Wacker P, Wacker R, Golnik R, Kreft HU (2003) Akute Lungenembolie: Ein neuer Score zur Quantifizierung der akuten Rechtsherzbelastung. Intensivmed 40:130-137 